\title{
Sisymbrium officinale, the Plant of Singers: A Review of Its Properties and Uses
}

\author{
Authors \\ Affiliations \\ 1 OU Endocrinology, Dept. Medicine (DIMED), University of \\ Padova, Padova, Italy \\ 2 Dipartimento di Scienze Biomediche, Università di Cagliari, \\ Cagliari, Italy \\ 3 AIROB, Associazione Italiana per la Ricerca Oncologica di \\ Base, Padova, Italy
}

Maira Zorzan ${ }^{1 *}$, Paolo Zucca ${ }^{2 *}$, Daniela Collazuol ${ }^{1}$, Stefania Peddio ${ }^{2}$, Antonio Rescigno ${ }^{2}$, Raffaele Pezzani ${ }^{1,3}$

Key words

Sisymbrium officinale, Erysimum officinale, hedge mustard, Erba dei cantanti, Hierba de los cantores, Brassicaceae

received November 15, 2019

revised December 27, 2019

accepted January 4, 2020

Bibliography

DOI https://doi.org/10.1055/a-1088-9928

published online February 4, 2020 | Planta Med 2020; 86:

307-311 @ Georg Thieme Verlag KG Stuttgart · New York |

ISSN 0032-0943

Correspondence

Raffaele Pezzani, DSc, MD, PhD,

OU Endocrinology, Department of Medicine (DIMED), University of Padova

Via Ospedale Civile 105, 35128 Padova, Italy

Phone: + 390498213018 , Fax: + 39049657391

raffaele.pezzani@unipd.it
Correspondence

Paolo Zucca, Prof, PhD

Dipartimento di Scienze Biomediche, Complesso Universitario

SP Monserrato-Sestu Km 0.70009042 Monserrato (CA)

Cagliari, Italy

Phone: + 390706754526 , Fax: + 390706754527

pzucca@unica.it

\section{ABSTRACT}

Sisymbrium officinale (hedge mustard and formerly called Erysimum officinale) is a common plant in wild lands of Europe and Africa. It is also cultivated for its seeds and leaves to be used in salad or mustard. Sisymbrium officinale is useful not only in culinary preparations, but it also seems to possess interesting therapeutic properties, especially for throat diseases such as aphonia and hoarseness. For this reason, it is commonly called "herb of singers" (in Italian, "Erba dei cantanti”). Indeed a cup of Sisymbrium officinale infusion is frequently consumed by singers before artistic performance, even if its beneficial ability still needs to be scientifically demonstrated. Some preliminary data can be analyzed, but new efforts and resources should be devoted to study and investigate a plant with valuable therapeutic potential. This review summarizes the data available for Sisymbrium officinale.

\section{Introduction}

Sisymbrium officinale (SO) (L.) Scop. (hedge mustard and formerly Erysimum officinale) is an annual herbaceous plant with a single stem erect or erect-branched at right angles, stiff and opaque green or purplish with scattered trichomes of $1 \mathrm{~mm}$ belonging to the Brassicaceae family ( $\triangleright$ Fig. 1 ). It features basal leaves petiolate, arranged in rosette, or deeply divided leaves with toothed margins and with a much larger rounded terminal lobe that grows up to $90 \mathrm{~cm}$ high. It is a native of Europe and North Africa, and it is now well-established throughout the world [1].

SO is a common weed of wasteland, roadsides, and cultivated and disturbed habitats. The basal (lower) leaves are up to $10 \mathrm{~cm}$ long, pinnatisect with 3-5 pairs of toothed lobes and a large terminal lobe petiolate. Middle and upper stem leaves are smaller, with or without a short petiole, much less divided and alternate. The stem is tall, erect, wiry, and with very short internodes. It is usually short-haired, sometimes glabrous. The small terminal flowers are in racemes with 4 yellow to pale yellow petals that are 2-4 mm long, in the shape of a cross. The inflorescence has no bracts. There are 4 free sepals that are $2 \mathrm{~mm}$ long, 4-6 stamens, the inner 4 being longer than the outer pair. The fruit is a siliqua with a peduncle close to the caule, erect of cylinder-conical shape 1-2 cm long, held close to the stem on a short stout pedicel (stalk) 1-2 mm long. At maturity, it opens along the 3 ribs releasing from each of the 2 valves, each with several seeds which measure about $1-1.5 \times 0.6 \times 0.8 \mathrm{~mm}[1,2]$.

These 2 authors should be regarded as co-first authors. 


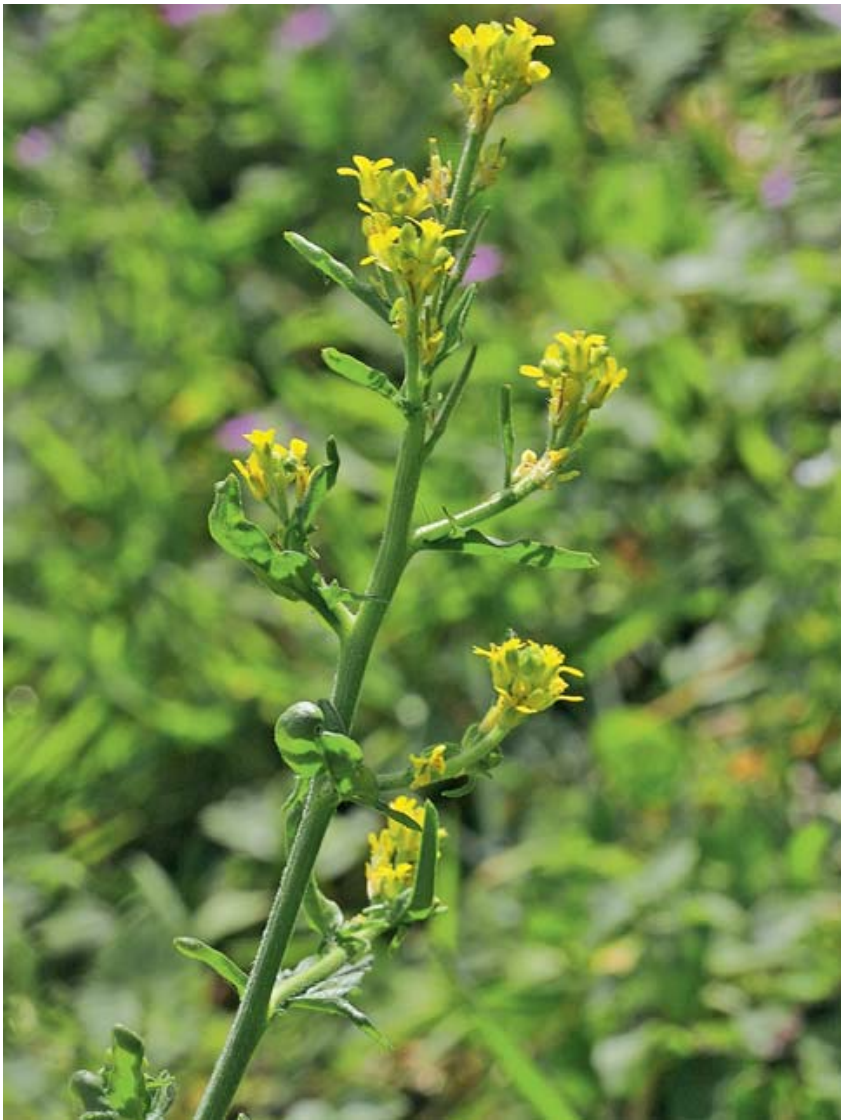

- Fig. 1 Sisymbrium officinale (L.) Scop. (hedge mustard) collected from Codoleddu, Cagliari, Italy.

Commonly called "the plant of singers" (in Spanish, "Hierba De Los Cantores"; in German, “Weg-Rauke or Gewöhnliche Rauke”), as its infusion is often ingested before an artistic performance. This plant is used for the treatment of respiratory tract diseases, such as pharyngitis, laryngitis, cough, aphonia, common cold, sore throat, and more rarely asthma [3]. It also shows antimicrobial, antimutagenic, and muscle relaxant activities, although these properties are less known and less exploited. Moreover, consumption of SO is suggested to smokers due to its anti-inflammatory and antioxidant effects [4]. It is of note that the use of SO was observed more than 2000 years ago in Greek and Roman times, when this plant as a practice was empirically used for treating many diseases, including mastitis, orchitis, and some tumors (without solid medical support) [5].

Despite several ethno-traditional indications on SO, clinical and scientific studies on its real biological activity are regrettably scarce. Very few articles investigating the potential therapeutic activities of SO are available in the most common medical and biological databases, such us "Embase-Elsevier", "Google Scholar", "PubMed", "Science Direct", "Web of Science", and "Scopus". Moreover, no human studies are available in the "ClinicalTrials. gov" database [6], the reference database for human clinical studies worldwide. The need to encourage such studies thus emerges, as well as the need to sensitize the decision-making centers toward concrete funding of biomedical research on this topic. On

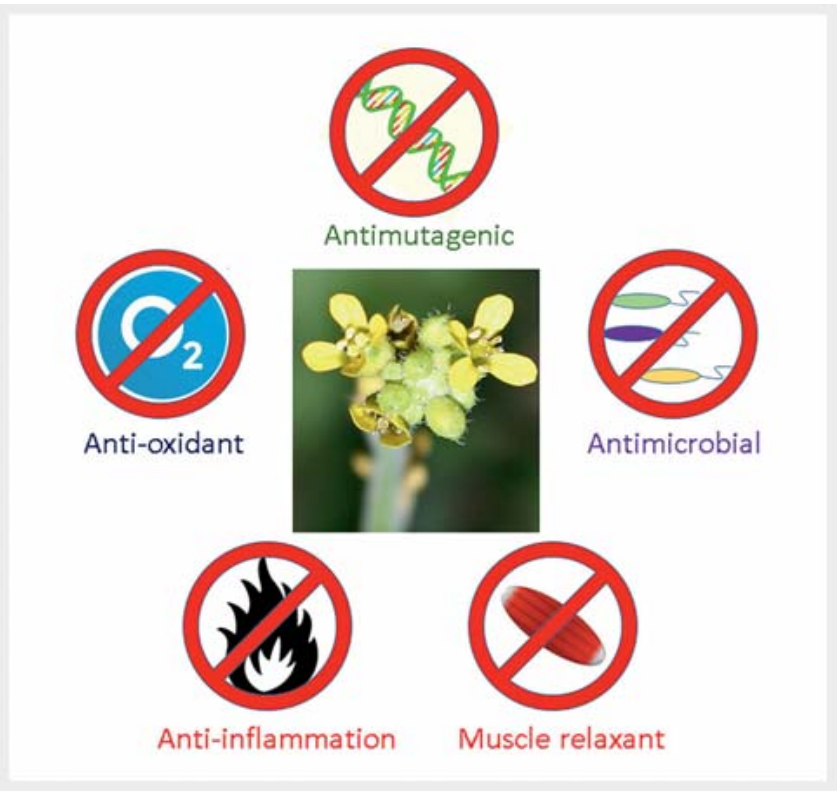

- Fig. 2 Schematic representation of the biological properties of Sisymbrium officinale.

the other hand, it is also important to make people aware of the lack of consistent and solid scientific data concerning the use of SO in humans, thus keeping in mind a precautionary principle whenever assuming a plant derivative. Nonetheless, a study reported in a non-indexed journal showed that a brief treatment with SO was effective in reducing the perceived disability of a group of subjects with vocal tract diseases [7]. These preliminary results need further confirmation, and the study is not present in the international bank "Clinical Trials"; however, the scientific community is gaining more and more awareness of the importance of finding scientific and rational answers to the consolidated and popular use of many plants or plant derivatives.

Despite the lack of clinical studies, there are some limited data, including the so-called ethno-traditional use and the preclinical studies, which can provide some indications on the properties of SO ( $\bullet$ Fig. 2 ), and these are summarized below in the next paragraphs.

\section{Phytochemistry}

From a pharmacological and biochemical point of view, the biological effects of SO can be mainly attributed to volatile compounds and particularly to the degradation products of glucosinolates, an important class of characteristic secondary metabolites present in many Angiosperms and especially in Brassicaceae [8, 9]. The most important glucosinolates of SO are all sulphated compounds and include glucoputranjivine, glucojiabutina, napoleiferina, sinalbina, and sinigrin [10]. Sulphated compounds, especially glucoputranjivine, are indeed the chemical markers of SO. Following rupture, cutting, or enzymatic digestion of the plant, the degradation of glucosinolates leads to their conversion into numerous derivatives, mainly isothiocyanates and nitriles, which possess numerous biological activities as above mentioned. In 
particular, the isothiocyanate seems to be the most active from a biological point of view, thanks to its activity against bacteria, fungi, nematodes, and insects, while nitriles seem to have more limited effects $[2,11]$.

The analysis of volatile compounds by gas chromatography coupled with mass spectrometry has allowed us to detect at least 42 different compounds, many of which have strong biological activities [12]. The chemical investigations on SO molecular composition showed that its air dried parts contain mucilages (10.9\%), ash $(9.2 \%)$, ictiols $(8.9 \%)$, glucosinolates $(0.63 \%)$, and flavonoids $(0.5 \%$, ) [13]. Among glucosinolates, isopropyl-glucosinolate represents about 65\% [8].

\section{SO Properties}

SO has been traditionally used for almost 2 millennia but only in the last decades has it been scientifically studied to confirm its properties. SO has been shown to possess different biological activities, which are summarized below.

\section{Antimicrobial activity}

The antimicrobial activity of SO has been tested on some bacterial and fungal strains. In particular, in 2010, Blazević and colleagues reported that the product of hedge mustard distillation in water inhibited the growth of several micro-organisms (5 species of Gram-positive bacteria and 9 Gram-negative ampicillin resistant bacterial species) [12]. Among the investigated bacterial strains, Gram-positive organisms, such as Clostridium perfringens, Enterococcus feacalis, and Micrococcus luteus, were the most affected species. A more moderate effect was observed on food pathogens such as Staphilococcus aureus and Bacillus cereus. Furthermore, ampicillin-resistant strains of Gram-negative bacteria were found to be sensitive to samples obtained from fresh plant material; in particular, these substances exhibited the highest activity against Chryseobacterium indologenes, Vibrio alginolyticus, and Aeromonas hydrophila. Among fungi, the most sensitive species included Aspergillus niger, Saccharomyces cerevisiae, and Penicillium sp [12]. In addition to this study, it should be noted that if the extraction method was applied to dried (not fresh) plant material, the extract had a lower inhibitory capacity on microorganisms, indicating a potential importance of the time between harvesting and its use and that the drying process itself induced some changes in the biochemistry of the plant that potentially could decrease its effectiveness [4]. Indeed, the volatile compounds derived from the degradation of glucosinolates decrease in concentration over time, and they are strongly involved in the antimicrobial effects of SO [12]. Not only glucosinolates are vital: alcohols, aldehydes, fatty acids, and corresponding esters had confirmed antimicrobial properties and could therefore contribute to the antimicrobial effect of the plant [12]. Unfortunately, in this paper, only the disc diffusion method has been applied: this approach (more straightforward) is typically used in the first stages of plant extract characterization [14], but confirmation with a more standardized and reliable method would be desirable. This consideration is even more important taking into account the fact that the only paper reporting an MIC determination of an aqueous SO extracts [15] showed no antimicrobial activity up to $1 \mathrm{mg} / \mathrm{mL}$. The tested strains were Gram-positive Staphylococcus aureus clinically isolated and Bacillus subtilis ATCC 6633; Gram-negative Escherichia coli and Pseudomonas cepacea clinically isolated, Shigella flexneri CDC9767, and Salmonella enteritidis IAL1132; and the yeast Candida albicans (clinically isolated). This is apparently in agreement with an observation made by Quave and colleagues about the inability of SO EtOH extract to inhibit the biofilm formation and the adherence of methicillin- resistant Staphylococcus aureus [16].

These conflicting data urge the need for proper and standardized comparison of different SO preparations (i.e., extraction with different solvent) to identify the actual presence of antimicrobial chemicals.

\section{Antimutagenic activity}

Glucosinolates and their degradation products have attracted considerable interest also for their potential cancer chemopreventive properties [17]. Some studies have shown that these compounds inhibited neoplastic process through various mechanisms, including the induction of apoptosis, blockade of mutagenesis, arrest of cell proliferation, and activation of epigenetic mechanisms [18]. In this regard, epidemiological studies suggest that the introduction of cruciferous vegetables in the diet consumption, as sources rich in glucosinolates, can reduce the risk of cancer and chronic degenerative diseases [19]. All studies analyzing the antimutagenic properties of SO were conducted on a reverse bacterial mutation assay, the so-called Ames test, applied to different Escherichia coli or Salmonella strains. The glucoputranjivine compound, as mentioned above [10], is the main glucosinolate found in the aerial parts of SO: it is considered the key responsible for the strong antimutagenic activity against a specific strain of Escherichia coli (WP2uvrA) and for the minor, but still present, activity against Salmonella typhimurium TA98 and TA100. The extract of SO was able to inhibit the mutagenic effect of potent carcinogens, such as 2-nitrofluorene, sodium azide, methyl methanesulfonate, and 2-aminoantracene $[4,15]$. Similarly, the antimutagenic activity was also studied by analyzing the glucosinolate metabolic derivatives, such as isopropylisothiocyanate and glucoputranjivine [20]. These derivatives were able to inhibit or prevent genotoxicity due to some mutagenic agents, such as methyl methanesulfonate, 2-aminoantracene, and 2-aminofluorene, and have been shown to inhibit carcinogenesis through deactivation of phase I enzymes and/or activation of phase II enzymes (cytosolic and microsomial enzymes involved in drug metabolism). Furthermore, the antimutagenic activity was tested both in the absence and in the presence of an exogenous metabolic activator, such as the $\mathbf{S} 9$ fraction system containing the cytochromes. It is indeed known that bacteria (unlike mammals) are unable to metabolize chemicals with cytochrome P450 and thus need an exogenous system that simulates the cellular environment typical of human cells. All these results suggest that the metabolic derivatives of glucoputranjivine have the ability to act alone and/or that they could be converted into biologically active derivatives, thanks to some non-specific degradation reactions that occur naturally in the cells [15]. Taken together these data support a role for $\mathrm{SO}$ in the protection of vocal cord as a traditional ethnobotanical use. 


\section{Antioxidant activity}

The antioxidant properties of SO have also been investigated through a biochemical system that can quantify the degree of inhibition of the damage produced by reactive oxygen species (ROS) following the use of an antioxidant substance. In fact, oxidative stress is the main vocal cord stress factor for tobacco smokers [15]. These studies showed an efficient "scavenging" ability of SO, which was therefore able to eliminate free radicals formed during the normal biochemical reactions [15]. Moreover, in this paper. the ability of SO extracts and polyphenol-enriched fractions to scavenge superoxide anion was first tested, showing IC50 ranging from 31.8 to $94.0 \mu \mathrm{g} / \mathrm{mL}$ (much higher than Trolox, a typical antioxidant standard, $822 \mu \mathrm{g} / \mathrm{mL}$ ). The authors also tested the inhibition of lipid peroxidation with IC50, in this case a little higher than the standard (111.2-153.5 $\mu \mathrm{g} / \mathrm{mL}$ vs. 47.6), and chelation and reduction of iron. In the latter case, only a polyphenolic fraction was able to chelate ferrous iron (such an activity is of great interest since it could be involved in the quenching Fenton-like ROS-generating reactions). The reported IC50 was similar to rutin, chosen as the standard. Unfortunately, to our knowledge, no antioxidant activity has been determined for SO extracts using commonly electron-transfer or hydrogen atom-transfer assays [21].

Furthermore, it has been observed that the radical quenching capacity was closely related to the antimutagenic activity described above: by limiting or preventing the formation of ROS, which often induce DNA breakdown and thus genetic mutations, SO can consequently also block the biochemical reactions potentially leading to damage of the genetic material. These potential antiradical and antioxidative activities can support the consumption of the plant by people working in highly polluted environments or even by smokers, who often suffer from problems related to the upper respiratory tract where SO seems to be traditionally more effective and whose ability to eliminate radicals is reduced compared to non-smokers [15,22].

\section{Anti-inflammatory activity}

Although less known, even the anti-inflammatory activity of SO could be at the basis of its marked antimicrobial and antimutagenic properties. Indeed it has been observed that the plant, containing adenine, adenosine, flavonoids, guanine, and oligosaccharides in the aqueous infusion, plays a mild anti-inflammatory action only at high concentrations, at least in the experimental topical animal model ("murine croton oil-induced ear edema model") [23]. The authors highlighted the necessity to strictly follow the traditional recipe $(2 \mathrm{~h}$ extraction of semi-fresh plant with hot water) to obtain a biological effect, whereas many papers dealt with organic extracts chemically and biologically different from traditional infusion.

Recently, Borgonovo and colleagues tried to identify a biological target responsible for anti-inflammatory activity of SO [9]. In this paper the transient receptor potential ankyrin 1 (TRPA1) channel has been targeted. This channel is involved in the somatosensory perception of pungency and in the nociception pathway of inflammatory pain. Two compounds in particular extracted from SO (isopropylisothiocyanate and 2-buthylisothiocyanate) proved to be potent agonists of TRPA1 (EC50 around $5 \mu \mathrm{M}$, being in the range of the high potency natural agonists up to now de- scribed). Also in this case, the authors stressed the importance of the procedure for infusion preparation, since it can deeply affect the biological activities of the samples.

No other works have analyzed the anti-inflammatory activity of SO; consequently its potential effects need to be amply verified in pharmacological studies, given its consolidated ethno-traditional use.

\section{Muscle relaxant activity}

One of the properties of the SO that can contribute to its use among singers is its potential muscle relaxant activity. There is only 1 study investigating this property of the plant, in which the efficacy of SO was tested in isolated guinea-pig trachea, used as an airway smooth muscle model. In this study, carbachol, histamine, and leukotriene C 4 were used as activators of contraction, and it evaluated the ability of SO to decrease the induced spasmodic action [4]. The authors showed that the plant could actually have a muscle relaxant effect and suggested that this scientific preliminary basis should be deeply and rapidly investigated.

\section{Conclusions and Future Perspectives}

The data obtained for the effectiveness of SO are not exhaustive and require further research studies. According to the EMA (European Medicines Agency), SO was traditionally used in medicine for a period of at least 30 years and therefore can be qualified as a traditional plant medicine according to the requirements reported in the Directive 2004/24/CE [24]. The EMA adds that the plant is a "Product to relieve throat irritation like hoarseness and dry cough". It also states that "since there are no clinical studies in the literature, its consolidated use cannot be recommended. Pharmacological studies on the anti-inflammatory and antimicrobial effects of SO could contribute to its traditional use; however, these effects have been reported only at high concentrations of the preparations or their components. The plausible effect of the plant on pharyngeal irritation could be due to the high content of mucilage (10.9-13.5\%)". The Agency draws further conclusions on the traditional use of SO: "As far as the aqueous extracts and their dosage are concerned, there are no safety problems due to the low content of cardenolide (cardiac steroid glycosides)", which could have potential cardiac effects in humans. The EMA also suggests that the plant should not be used in children under 6 years of age, and during pregnancy and lactation, as there are no studies on these groups of risk. Furthermore, "due to the lack of genotoxicity data, the inclusion of SO in the European List of herbal substances, their preparations or combinations to be used in traditional medicinal products cannot be recommended" [24]. Therefore, it can be argued that the European Agency, due to the lack of clinical studies on humans, is adopting a precautionary principle (that the authors shared), although it underlines that the consolidated ethno-traditional use of SO could show a certain efficacy.

Again, 2 fundamental aims emerge as necessary and urgent: the set-up of controlled and randomized clinical trials to investigate the efficacy of SO in diseases of the upper airways, and, on the other hand, research based on pre-clinical studies to deeper investigate and characterize the mechanism of action of SO in or- 
der to decisively find a scientific origin for plant effects. To date, indeed, pre-clinical studies are poorly represented, sectorial, and lack more significant pharmacological data. Many efforts are still necessary to transform the ethno-traditional use of SO into a concrete therapeutic option.

Acknowledgements

We wish to thank Mr. Giuliano Campus (www.sardegnaflora.it) for kindly providing the image of the Sysimbrium officinale.

\section{Conflict of Interest}

The authors declare that they have no conflict of interest.

\section{References}

[1] Pignatti S. Flora d'Italia. Bologna: Edagricole; 1982

[2] Leclerc H. Précis de Phytothérapie. Paris; Elsevier Masson; 1983

[3] Rahman M, Khatun A, Liu L, Barkla B]. Brassicaceae mustards: traditional and agronomic uses in Australia and New Zealand. Molecules 2018; 23: E231

[4] Di Sotto A, Vitalone A, Nicoletti M, Piccin A, Mazzanti G. Pharmacological and phytochemical study on a Sisymbrium officinale Scop. extract. J Ethnopharmacol 2010; 127: 731-736

[5] Karpozilos A, Pavlidis N. The treatment of cancer in Greek antiquity. Eur J Cancer 2004; 40: 2033-2040

[6] ClinicalTrials.gov. Clinical Trials. Available at https://clinicaltrials.gov/. Accessed January 31, 2020

[7] Calcinoni O. Sisymbrium "Singers' plant" efficacy in reducing perceived vocal tract disability. J Otolaryngology-ENT Res 2017. doi:10.15406/ joentr.2017.08.00243

[8] Griffiths DW, Deighton N, Birch AN, Patrian B, Baur R, Stadler E. Identification of glucosinolates on the leaf surface of plants from the Cruciferae and other closely related species. Phytochemistry 2001; 57: 693-700

[9] Borgonovo G, Zimbaldi N, Guarise M, De Nisi P, De Petrocellis L, Schiano Moriello A, Bassoli A. Isothiocyanates and glucosinolates from Sisymbrium officinale (L.) Scop. ("the Singers' Plant"): isolation and in vitro assays on the somatosensory and pain receptor TRPA1 channel. Molecules 2019; 24: E949

[10] Fahey JW, Zalcmann AT, Talalay P. The chemical diversity and distribution of glucosinolates and isothiocyanates among plants. Phytochemistry 2001; 56: 5-51
[11] Guarise MB, Borgonovo G, Bassoli A, Ferrante A. Evaluation of two wild populations of hedge mustard (Sisymbrium officinale (L.) Scop.) as a potential leafy vegetable. Horticulturae 2019; 5: 13

[12] Blazevic I, Radonic A, Mastelic ], Zekic M, Skocibusic M, Maravic A. Hedge mustard (Sisymbrium officinale): chemical diversity of volatiles and their antimicrobial activity. Chem Biodivers 2010; 7: 2023-2034

[13] Carnat AF, Fraisse D, Carnat AP, Groubert A, Lamaison JL. Normalisation de l'erysimum, Sisymbrium officinale L. Ann Pharm Fr 1998; 56: 36-39

[14] Zucca P, Bellot S, Rescigno A. The modern use of an ancient plant: exploring the antioxidant and nutraceutical potential of the Maltese mushroom (Cynomorium Coccineum L.). Antioxidants 2019; 8: E289

[15] Di Sotto A, Di Giacomo S, Toniolo C, Nicoletti M, Mazzanti G. Sisymbrium officinale (L.) Scop. and its polyphenolic fractions inhibit the mutagenicity of tert-butylhydroperoxide in Escherichia coli WP2uvrAR strain. Phytother Res 2016; 30: 829-834

[16] Quave CL, Plano LR, Pantuso T, Bennett BC. Effects of extracts from Italian medicinal plants on planktonic growth, biofilm formation and adherence of methicillin-resistant Staphylococcus aureus. J Ethnopharmacol 2008; 118: 418-428

[17] Capuano E, Dekker M, Verkerk R, Oliviero T. Food as pharma? The case of glucosinolates. Curr Pharm Des 2017; 23: 2697-2721

[18] Higdon JV, Delage B, Williams DE, Dashwood RH. Cruciferous vegetables and human cancer risk: epidemiologic evidence and mechanistic basis. Pharmacol Res 2007; 55: 224-236

[19] Verhoeven DT, Goldbohm RA, van Poppel G, Verhagen H, van den Brandt PA. Epidemiological studies on brassica vegetables and cancer risk. Cancer Epidemiol Biomarkers Prev 1996; 5: 733-748

[20] Di Sotto A, Di Giacomo S, Vitalone A, Nicoletti M, Mazzanti G. Antimutagenic thio compounds from Sisymbrium officinale. J Nat Prod 2012; 75: 2062-2068

[21] Attia IBZ, Zucca P, Marincola FC, Piras A, Rosa A, Chaieb M, Rescigno A. Chemical composition and antioxidant potential differences between Cynomorium coccineum L. Growing in Italy and in Tunisia: effect of environmental stress. Diversity (Basel) 2018; 10: 53

[22] Boni UP, Patri G. Le erbe: medicinali aromatiche cosmetiche. Milano, Italy: Fratelli Fabbri; 1976

[23] Politi MB, Braca A, Altinier G, Sosa S, Ndjoko KI, Wolfender JL, Hostettmann $\mathrm{K}$, jiménez-Barbero J. Different approaches to study the traditional remedy of "hierba del canto", Sisymbrium officinale (L.) Scop. Boletín Latinoamericano y del Caribe de Plantas Medicinales y Aromáticas 2008; 7: 30-37

[24] Committee on Herbal Medicinal Products. Assessment report on Sisymbrium officinale (L.) Scop., herba. EMA/HMPC/280195/2013 